\title{
Article
}

\section{Écobilans de biocarburants : une revue des controverses}

\author{
Bruno Dorin, Vincent Gitz \\ Économistes, CIRAD, UMR Cired, 34398 Montpellier, France
}

\begin{abstract}
Les débats autour des biocarburants, un temps présentés comme une solution à la raréfaction du pétrole et à la réduction d'émission de gaz à effet de serre, s'intensifient. Le texte que nous publions éclaire ces débats en comparant les résultats des quelques écobilans disponibles concernant la production des biocarburants. L'analyse du cycle de vie (ACV) est la méthode privilégiée par le monde industriel pour apprécier l'impact environnemental. Il souffre de nombreuses imperfections, tout comme le calcul de l'empreinte écologique chère aux ONG et, de manière générale, comme tous les indicateurs dits du « développement durable ». Aussi, au-delà de la comparaison des divers écobilans, cet article introduit la question des méthodologies à mettre en œuvre pour l'évaluation des coûts et bénéfices de production de biocarburants au regard des enjeux agronomiques mondiaux.
\end{abstract}

La Rédaction

\section{Mots-clés :}

agriculture ; biocarburant ; écobilan; $\mathrm{ACV}$

\section{Keywords:}

agriculture; biofuels; bioenergy; energy crops; LCA

\begin{abstract}
Résumé - Cette revue d'écobilans de biocarburants synthétise un ensemble de questions soulevées par l'émergence de la production de biocarburants et la place ambitionnée pour ces derniers dans les consommations énergétiques à venir. Elle montre également que les écobilans réalisés à ce jour doivent être approfondis et harmonisés sur certains aspects méthodologiques, et qu'ils demeurent restreints en nombre tout en étant largement centrés sur quelques agro-industries nord-américaines et ouest-européennes. Il serait bénéfique d'élargir ces analyses à d'autres contextes, biomasses et modes agricoles de production pour deux grandes raisons : (i) la recherche de réservoirs durables de bioénergies l'impose, (ii) le résultat des écobilans s'avère fortement sensible, d'une part aux techniques agricoles employées (motorisation, intrants chimiques, irrigation) dans des écosystèmes spécifiques (types de climat et de sol), d'autre part aux arbitrages relatifs à la valorisation des coproduits (alimentation animale, amélioration des sols, coproduction d'énergie).
\end{abstract}

\begin{abstract}
Life-Cycle-Analyses of biofuels: a review of controversies. This review of Life-CycleAnalyses of biofuels synthesizes a wide range of questions raised by the increasing production of biofuels and by agendas aimed at boosting biofuels in future energy consumption. It also shows that current LCAs must be deepened or harmonized in some methodological aspects, and that they remain restricted in number as well as focused on only few North-American and West-European agro-industries. Expanding these analyses to other contexts, biomasses and agricultural modes of production would prove beneficial chiefly for two reasons: (1) there are almost no other ways of exploring and identifying sustainable reservoirs of bioenergies, (2) the LCA results turn out to be highly susceptible on the one hand to the agricultural techniques applied (motorization, chemical inputs, irrigation) in specific ecosystems (climate and soil types), on the other hand to arbitrations relating to the use of co-products (animal feed, soil enhancement, co-production of energy).
\end{abstract}

\section{Introduction}

Au milieu des années 2000, l'Agence internationale de l'énergie estime qu'environ $1 \%$ des surfaces cultivées sont consacrées à la production de biocarburants (liquides) et que ceux-ci substituent dans une proportion similaire notre consommation mondiale de carburants fossiles. À

Auteur correspondant : B. Dorin, bruno.dorin@cirad.fr l'avenir, cette part pourrait considérablement augmenter ${ }^{1}$.

\footnotetext{
${ }^{1}$ Selon le GIEC, les bioénergies au sens large pourraient techniquement générer $441 \mathrm{EJ} \mathrm{an}^{-1}$ à l'horizon 2050 (IPCC, 2001a, 244), voire beaucoup plus (Smeets et Faaij, 2007, Smeets et al., 2007), alors qu'en 2002, elles ne fournissent que $46 \mathrm{EJ}$ (13,4\% de l'approvisionnement en énergie primaire), dont $70 \%$ sous forme de bois, charbon de bois, résidus de récolte et bouses abondamment utilisés tels quels dans les pays du Sud (Sims
} et al., 2006). 
Tableau 1. Avantages et inconvénients apparents des biocarburants.

\begin{tabular}{|c|c|c|}
\hline & Avantages apparents & Inconvénients apparents \\
\hline $\begin{array}{l}\text { Approvisionnent } \\
\text { énergétique }\end{array}$ & $\begin{array}{l}\text { - Réduction des factures pétrolières } \\
\text { - Diversification et décentralisation des approvi- } \\
\text { sionnements énergétiques }\end{array}$ & $\begin{array}{l}\text { - Coûts de production et de distribution plus } \\
\text { élevés que les hydrocarbures }\end{array}$ \\
\hline $\begin{array}{l}\text { Agriculture } \\
\text { Alimentation }\end{array}$ & $\begin{array}{l}\text { - Maintien/Amélioration des revenus et/ou em- } \\
\text { plois agricoles }\end{array}$ & $\begin{array}{l}\text { - Pression inflationniste sur le prix des produits } \\
\text { agricoles et alimentaires }\end{array}$ \\
\hline Industrie & $\begin{array}{l}\text { - Hausse de l'indice d'octane (éthanol) ou de } \\
\text { cétane (biodiesel), meilleure viscosité (biodiesel) } \\
\text { - Facilité d'utilisation (adaptation plus onéreuse } \\
\text { des véhicules et/ou infrastructures au gaz ou à } \\
\text { l'électricité) }\end{array}$ & $\begin{array}{l}\text { - Moindre pouvoir calorifique } \\
\text { - Adaptation nécessaire des véhicules, carburants } \\
\text { et/ou infrastructures au-delà de certains seuils } \\
\text { d'incorporation, notamment (éthanol) pour palier } \\
\text { l'instabilité du mélange avec l'eau, la corrosion } \\
\text { plus rapide de certains métaux, l'augmentation de } \\
\text { la volatilité et des risques associés }\end{array}$ \\
\hline Environnement & $\begin{array}{l}\text { - Réduction des émissions de GES } \\
\text { - Réduction des émissions de } \mathrm{CO}, \mathrm{HC} \text {, particules } \\
\text { solides (éthanol, biodiesel), } \mathrm{SO}_{2} \text {, benzène, buta- } \\
\text { diène, isobutène, toluène, xylène (éthanol), souffre, } \\
\text { composés aromatiques (biodiesel) } \\
\text { - Valorisation de déchets ou sous-produits : résidus } \\
\text { de récolte, déchets forestiers ou municipaux, petit- } \\
\text { lait, suif, huiles ou graisses alimentaires... }\end{array}$ & $\begin{array}{l}\text { - Maintien/Accroissement des pollutions par les } \\
\text { intrants agricoles, notamment de } \mathrm{N}_{2} \mathrm{O} \text { par les } \\
\text { engrais azotés } \\
\text { - Augmentation des émissions de } \mathrm{NO}_{x} \text { (éthanol, } \\
\text { biodiesel), composés organiques volatiles, acétal- } \\
\text { déhydes, PAN (éthanol) } \\
\text { - Augmentation possible des surfaces cultivées au } \\
\text { détriment d'autres espaces (forêts, jachères...) }\end{array}$ \\
\hline
\end{tabular}

Sources : d'après CES (2004) et IEA (2004), essentiellement.

D'ambitieux plans sont pour cela mis en œuvre au Brésil, aux États-Unis, ou encore en Europe sur la base d'un engagement fort des États (Tréguer, 2007). La fourniture d'énergie d'origine agricole et forestière n'est pas nouvelle depuis la révolution industrielle (Fernandes et al., 2007), mais pour les biocarburants, des facteurs de diverses natures $^{2}$ ont encouragé puis découragé leur développement depuis leur première expérimentation au XIX ${ }^{\mathrm{e}}$ siècle en France (éthanol de betterave) et en Allemagne (huiles végétales). Parmi leurs bénéfices apparents (Tab. 1) (deux sont désormais avancés pour légitimer et encourager une croissance plus soutenue de leur production ici et là dans le monde : (i) réduire la dépendance des économies aux énergies fossiles, (ii) atténuer les émissions anthropiques de gaz à effet de serre (GES).

Dans la communauté scientifique cependant, et comme nous allons le montrer, ces deux points sont encore débattus. La plupart des auteurs impliqués mobilise pourtant une même méthodologie, celle de l'analyse de cycle de vie $(\mathrm{ACV})$. Cette dernière, aussi appelée « écobilan » ${ }^{3}$, est apparue dans les années 1970 et s'utilise de plus en plus couramment dans l'industrie depuis l'apparition des normes ISO 14040. Elle est également l'objet d'une revue scientifique : The International Journal

\footnotetext{
${ }^{2}$ Essor de la pétrochimie, accroissement de la population mondiale et des besoins alimentaires, crises et contre-chocs pétroliers, politiques nationales de soutien des revenus agricoles, de lutte contre la pollution de l'air, etc.

${ }^{3} \mathrm{~S}^{\prime}$ il existe des différences de définition entre ACV et écobilan, elles ne sont pas l'objet d'un net consensus.
}

of Life Cycle Assessment. Elle vise à évaluer (en particulier) les impacts environnementaux d'un produit, d'un service ou d'un procédé, ceci de leur « berceau » à leur « tombe » (cradle-to-grave) ou, dans les cas rapportés ci-après, du champ (puits) au réservoir (well-to-tank) ou à la roue (well-to-wheel). La première étape $\mathrm{d}$ 'une $\mathrm{ACV}$ consiste d'abord à définir les objectifs et le champ de l'étude ; on inventorie ensuite les flux (de matières, d'énergies, de services...) qui entrent et sortent du système considéré, puis après traduction de ces derniers en unités appropriées aux objectifs, on procède à l'interprétation des résultats.

Basé sur une revue assez large de la littérature, cet article poursuit un triple objectif ${ }^{4}$ : (i) rapporter les résultats d'ACV de biocarburants et expliquer leurs divergences relatives à l'économie d'énergie ; (ii) procéder de même pour les émissions de GES, (iii) élargir le débat sur les $\mathrm{ACV}$ et leur capacité à révéler les enjeux et arbitrages auxquels sont aujourd'hui exposées les agricultures du Nord et du Sud ${ }^{5}$.

\footnotetext{
4 Cet article a été élaboré dans le cadre du projet «Compétition énergie-alimentation dans l'usage des sols : perspectives et viabilité des biocarburants » développé avec un appui financier du Conseil français de l'énergie (CFE). Il n'engage que ses auteurs et non leur institution ou bailleur de fonds.

${ }^{5}$ La partition Nord/Sud que nous entérinons ici par facilité d'écriture est assurément réductrice et par là trompeuse ; par "Sud", nous entendons néanmoins un relatif rapprochement en termes d'écosystèmes (tropicaux et subtropicaux) et de revenus (faibles par tête), ceci comparé aux pays du «Nord »...
} 
Tableau 2. Bilan énergétique des carburants et biocarburants selon l'ADEME.

\begin{tabular}{|c|c|c|c|c|}
\hline (mégajoules utilisés par mégajoules fournis) & $\begin{array}{c}\text { Pétrole } \\
\downarrow \\
\text { Essence, } \\
\text { MTBE } \\
\end{array}$ & $\begin{array}{c}\text { Pétrole } \\
\quad \downarrow \\
\text { Gazole }\end{array}$ & $\begin{array}{l}\text { Blé Betterave } \\
\qquad \downarrow \\
\text { Éthanol, ETBE }\end{array}$ & $\begin{array}{c}\text { Colza } \\
\text { Tournesol } \\
\downarrow \\
\text { Huile, EMHV } \\
\end{array}$ \\
\hline $\begin{array}{l}\text { Carburant « simple» (essence, gazole, éthanol ou } \\
\text { huile) }\end{array}$ & 1,15 & 1,09 & 0,49 & 0,20 \\
\hline Carburant «élaboré » (MTBE, ETBE ou EMHV) & 1,32 & - & 0,98 & 0,33 \\
\hline \multicolumn{5}{|l|}{ Répartition par phases: } \\
\hline - Culture ou extraction & 0,35 & 0,55 & 0,10 & 0,14 \\
\hline $\begin{array}{l}-1^{\text {re }} \text { transformation (en essence, gazole, éthanol ou } \\
\text { huile) }\end{array}$ & 0,69 & 0,44 & 0,39 & 0,04 \\
\hline $\begin{array}{l}-2^{e} \text { transformation éventuelle (en MTBE, ETBE ou } \\
\text { EMHV) }\end{array}$ & 0,17 & - & 0,49 & 0,13 \\
\hline - Transport & 0,12 & 0,11 & $(<5 \%)$ & $(<5 \%)$ \\
\hline
\end{tabular}

Source : d'après ADEME et al. (2002).

Notes : le MTBE (Methyl-Tertio-Butyl-Ether) est un composé oxygéné dérivé du méthanol, l'ETBE (Ethyl-Tertio-Butyl-Ether) combine l'éthanol (47\%) avec l'isobutylène (53\%), et l'EMHV est l'ester méthylique d'huile végétale.

\section{La consommation énergétique}

En première approche, les biocarburants rétrocèdent sous forme liquide de l'énergie solaire captée par photosynthèse durant leur fabrication, et non de l'énergie fossile comme l'essence ou le diesel. Cette économie d'énergie fossile doit cependant être minorée par celle qui est mobilisée directement (via tracteurs, camions, usines...) et indirectement (fabrication d'engrais, pesticides, électricité...) pour produire et distribuer le biocarburant. L'ACV offre un cadre pour évaluer cette énergie consommée en amont $\left(E_{\text {in }}\right)$, rapporter cette dernière à l'énergie fournie en aval $\left(E_{\text {out }}\right)$, et comparer le ratio d'efficacité énergétique ainsi obtenu $\left(I=E_{\text {in }} / E_{\text {out }}\right)$ avec celui de l'essence ou du diesel. Ainsi, pour ADEME et al. (2002), $I=0,49$ pour l'éthanol français de blé ou de betterave contre 1,15 pour l'essence, et $I=0,33$ pour l'ester de colza ou de tournesol contre 1,09 pour le gazole (Tab. 2). À service égal, les biocarburants économiseraient donc de substantielles quantités d'énergie fossile (57 et $70 \%$ en l'occurrence). Divers autres auteurs confirment ces économies, mais dans des mesures généralement plus modestes, et exception faite de Pimentel et Patzek qui concluent que l'essentiel des biocarburants ont un rendement énergétique désastreux (Tab. 3). Au-delà des vives controverses que ces derniers auteurs ont suscité, controverses que certains jugent inutiles non sans arguments ${ }^{6}$, ces écarts plus ou moins importants de résultats s'avèrent pour

${ }^{6}$ En reprenant des estimations de Farrell et al. (2006, Fig. 2), Dale (in AI, 2006, 31-41) déduit en effet qu'en comparaison de l'essence, l'éthanol de maïs américain permet de diviser par 22 la dépendance de la production de carburant au pétrole, puisque ce biocarburant mobilise avant tout pour sa fabrication d'autres formes d'énergie fossile (charbon et gaz en l'occurrence).

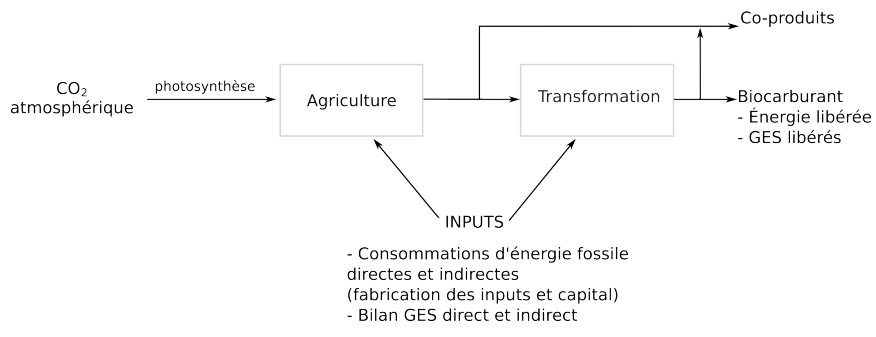

Fig. 1. Processus de fabrication des biocarburants.

beaucoup reposer sur des choix divergents relatifs, d'une part aux coproduits, d'autre part au périmètre d'analyse.

La production des biocarburants peut en effet générer un ou plusieurs coproduits (Fig. 1) valorisés en alimentation animale (tourteau d'oléagineux, drèche de céréale, vinasse...) ou ailleurs (glycérine, électricité...). L'énergie entrée dans le processus $\left(E_{\text {in }}\right)$ ne sert donc pas à produire que du biocarburant. Pour le calcul de $I$, il faut alors déduire a priori de $E_{\mathrm{in}}$ la fraction d'énergie mobilisée pour fabriquer ces coproduits. Cette fraction étant généralement inconnue, l'ADEME a opté pour une méthode d'allocation simple - dite "massique » - consistant à répartir $E_{\text {in }}$ au prorata des masses de produits obtenus en sortie. Quand la masse de coproduits est importante, cela revient donc à affecter à ces derniers l'essentiel de $E_{\text {in }}$ et à améliorer d'autant l'efficacité énergétique I du biocarburant. Or selon Sourie et al. (2005) ou Sadones (2006), cette méthode n'est acceptable qu'en pétrochimie où les produits obtenus sont de finalité et de densité énergétique similaires. De fait, la grande majorité des ACV de biocarburants optent pour d'autres méthodes d'allocation de $E_{\text {in }}$. Chacune d'elles est accompagnée d'un lot de critiques (Farrell et al., 2006; 
Tableau 3. Bilan énergétique et bilan effet de serre des biocarburants de $1^{\text {re }}$ génération.

\begin{tabular}{|c|c|c|c|c|c|c|}
\hline Biomasse & Auteurs & Année & Région & [1] & [2] & [3] \\
\hline \multicolumn{7}{|l|}{ 1. Éthanol } \\
\hline Maïs & GMC et al. (2001) in IEA (2004) & 2001 & USA & $0,50-0,55(54 \%)$ & & \multirow{11}{*}{$\begin{array}{c}3100 \\
\text { (USA, c.2002) }\end{array}$} \\
\hline \multirow[t]{10}{*}{ (grain) } & Wang in IEA (2004) & 2001 & USA & $0,54-0,57(52 \%)$ & $32-25 \%$ & \\
\hline & Shapouri (2004) & 2004 & USA & $0,57-0,64(47 \%)$ & & \\
\hline & Levellon in IEA (2004) & 2000 & Canada & $0,67(42 \%)$ & $38 \%$ & \\
\hline & Marland in IEA (2004) & 1991 & USA & $0,78(32 \%)$ & $21 \%$ & \\
\hline & Hill et al. (2006) Tilman et al. (2006) & 2006 & USA & $0,80(30 \%)$ & $12 \%$ & \\
\hline & Graboski (2002) & 2002 & USA & $0,83(28 \%)$ & & \\
\hline & Levy in CONCAWE (2002) in IEA (2004) & 1993 & UE & $0,85-0,95(22 \%)$ & $33-30 \%$ & \\
\hline & Oliveira et al. (2005) & 2005 & USA & $0,91(21 \%)$ & & \\
\hline & Pimentel et Patzek (2005) & 2005 & USA & $1,29(-12 \%)$ & & \\
\hline & Pimentel, 2001, in IEA (2004) & 2001 & USA & $1,65(-43 \%)$ & $-30 \%$ & \\
\hline \multirow{11}{*}{$\begin{array}{l}\text { Blé } \\
\text { (grain) }\end{array}$} & Elsayed et al. (2003) avec références 1996 & 2003 & UE & $0,43-0,50(60 \%)$ & & \multirow{11}{*}{$\begin{array}{c}2500 \\
\text { (UE, c.2002) }\end{array}$} \\
\hline & ADEME et al. (2002) & 2002 & UE & $0,49(57 \%)$ & $60 \%$ & \\
\hline & Levy in CONCAWE (2002) in IEA (2004) & 1993 & UE & $0,81(30 \%)$ & $32-35 \%$ & \\
\hline & Sourie et al. (2005) & 2005 & UE & $0,84(27 \%)$ & & \\
\hline & Richards (2000) in CONCAWE (2002) in IEA (2004) & 2000 & UE & $0,90(22 \%)$ & $29 \%$ & \\
\hline & Punter et al. (2004) & 2004 & UE & $0,90(22 \%)$ & $7-37 \%$ & \\
\hline & ETSU in CONCAWE (2002) in IEA (2004) & 1996 & UE & $0,98(15 \%)$ & $47 \%$ & \\
\hline & Woods et Bauen (2003) & 2003 & UE & $0,60-1,45(11 \%)$ & & \\
\hline & UE in CONCAWE (2002) in IEA (2004) & 1994 & UE & $1,03(10 \%)$ & $19 \%$ & \\
\hline & Gover et al. in Jeuland et al. (2004) & 1996 & UE & $1,07(7 \%)$ & $41 \%$ & \\
\hline & UE in Jeuland et al. (2004) & 1994 & UE & $1,26(-10 \%)$ & $1 \%$ & \\
\hline Betterave & ADEME et al. (2002) & 2002 & UE & $0,49(57 \%)$ & $61 \%$ & \multirow{8}{*}{$\begin{array}{c}5500 \\
\text { (UE, c.2002) }\end{array}$} \\
\hline \multirow[t]{7}{*}{ à sucre } & Elsayed et al. (2003) à partir de Kaitschmitt et al. (1997) & 2003 & UE & $0,45-0,54(57 \%)$ & & \\
\hline & UE in CONCAWE (2002) in IEA (2004) & 1994 & UE & $0,64(44 \%)$ & $50 \%$ & \\
\hline & GM (2002) in IEA (2004) & 2002 & UE & $0,65(43 \%)$ & $41 \%$ & \\
\hline & Woods et Bauen (2003) & 2003 & UE & $0,62-0,74(41 \%)$ & & \\
\hline & Levy in CONCAWE (2002) in IEA (2004) & 1993 & UE & $0,56-0,84(39 \%)$ & $56-35 \%$ & \\
\hline & Sourie et al. (2005) & 2005 & UE & $0,78(32 \%)$ & & \\
\hline & UE in Jeulan et al. (2004) & 1994 & UE & $0,96(17 \%)$ & $25 \%$ & \\
\hline Canne & Macedo et al. (2002) in IEA (2004) & 2002 & Brésil & $0,12(90 \%)$ & $92 \%$ & \multirow{4}{*}{$\begin{array}{c}6500 \\
\text { (Brésil, c.2002) }\end{array}$} \\
\hline \multirow[t]{3}{*}{ à sucre } & Oliveira et al. (2005) & 2005 & Brésil & $0,27(77 \%)$ & & \\
\hline & Pimentel et Patzek (2007) & 2007 & Brésil & $0,72(37 \%)$ & & \\
\hline & Pimentel et Patzek (2007) & 2007 & USA & $0,89(23 \%)$ & & \\
\hline \multicolumn{7}{|l|}{ 2. Biodiesel } \\
\hline Colza & ADEME et al. (2002) & 2002 & UE & $0,33(70 \%)$ & $78 \%$ & \multirow{8}{*}{$\begin{array}{c}1200 \\
(\mathrm{UE}, \mathrm{c} .2002)\end{array}$} \\
\hline \multirow[t]{7}{*}{ (grain) } & GM (2002) in IEA (2004) & 2002 & UE & $0,33(70 \%)$ & $49 \%$ & \\
\hline & Richards (2000) in CONCAWE (2002) in IEA (2004) & 2000 & UE & $0,40(63 \%)$ & $58 \%$ & \\
\hline & Elsayed et al. (2003) à partir de Kaitschmitt et al. (1997) & 2003 & UE & $0,41-0,46(60 \%)$ & & \\
\hline & Sharmer in CONCAWE (2002) in IEA (2004) & 1996 & UE & $0,41-0,55(56 \%)$ & $66-56 \%$ & \\
\hline & Woods et Bauen (2003) & 2003 & UE & $0,53(51 \%)$ & & \\
\hline & Levy in CONCAWE (2002) in IEA (2004) & 1993 & UE & $0,52-0,57(50 \%)$ & $48-44 \%$ & \\
\hline & ETSU in CONCAWE (2002) in IEA (2004) & 1996 & UE & $0,82(26 \%)$ & $56 \%$ & \\
\hline \multirow{2}{*}{$\begin{array}{l}\text { Tournesol } \\
\text { (grain) }\end{array}$} & ADEME et al. (2002) & 2002 & UE & $0,32(71 \%)$ & $83 \%$ & \multirow{2}{*}{$\begin{array}{c}1000 \\
\text { (UE, c.2002) }\end{array}$} \\
\hline & Pimentel et Patzek (2005) & 2005 & USA & $1,96-2,18(-90 \%)$ & & \\
\hline \multirow{2}{*}{$\begin{array}{l}\text { Soja } \\
\text { (fève) }\end{array}$} & Hill et al. (2006) Tilman et al. (2006) & 2006 & USA & $0,52(52 \%)$ & $41 \%$ & \multirow{2}{*}{$\begin{array}{c}500 \\
\text { (USA, c.2002) }\end{array}$} \\
\hline & Pimentel et Patzek (2005) & 2005 & USA & $1,08-1,27(-8 \%)$ & & \\
\hline Palmier & Azevedo et Kaltner in WWI (2006) & $?$ & $?$ & $0,11(90 \%)$ & & \\
\hline
\end{tabular}

Légende :

[1] Énergie dépensée/énergie fournie, du champ à la roue en règle générale (entre parenthèses : économie d'énergie par rapport à un ratio de 1,15 pour l'essence et 1,09 pour le gazole d'après ADEME, 2002).

[2] Réduction d'émission de $\mathrm{GES}\left(\mathrm{CO}_{2}, \mathrm{~N}_{2} \mathrm{O}\right.$ et $\mathrm{CH}_{4}$ en $\left.\mathrm{E}_{\mathrm{q}} \mathrm{CO}_{2}\right)$ par kilomètre parcouru, par rapport à un véhicule « classique » (essence ou diesel selon les cas), du champ à la roue en règle générale.

[3] Rendement typique en litre de biocarburant par hectare (circa 2002) d'après IEA (2004, 127), aux États-Unis (USA), Union Européenne (UE) ou Brésil. 
Hecht, 2007; Kavanagh, 2006 ; Larson, 2005 ; Shapouri, 2002, etc.), qu'elles se basent sur le contenu énergétique des coproduits (pouvoir calorifique, calories alimentaires), les résultats d'une modélisation thermodynamique du procédé industriel (type Aspen Plus), les valeurs de marché des coproduits ou leur valeur de remplacement par un substitut, l'énergie dépensée pour produire un substitut (par exemple du tourteau de soja pour drèche) ou pour remplacer le coproduit par un substitut. On peut enfin et aussi, comme l'argumentent Patzek ou encore Woods et Bauen (2003), n'accorder aucun « crédit énergétique» aux coproduits. Ces divergences méthodologiques fournissent une première explication à la variabilité des résultats (Tab. 3), résultats tendant néanmoins à montrer que l'efficacité énergétique des biodiesels surpasse celle des actuels éthanols de céréales : ces derniers valorisent encore mal leurs coproduits dans l'alimentation animale, ou dans la cogénération d'énergie à l'instar de l'éthanol brésilien de canne à sucre avec la bagasse (WADE, 2004).

Concernant le périmètre d'analyse, dans les travaux de l'ADEME (2002) comme dans ceux de Shapouri (2004) et bien d'autres, il est précisé que les calculs n'intègrent par exemple pas les flux d'énergie fossile dépensée pour produire des facteurs fixes de production tels qu'équipements et bâtiments agricoles. Pour Pimentel et Patzek (2005), et plus modérément Delucci (2004), il s'agit là d'une importante lacune car dans la production américaine de maïs par exemple, une abondance de tracteurs, semoirs, épandeurs, moissonneuses et autres équipements sont mobilisés. Shapouri avait argumenté en 2004 : « l'information dans ce domaine est ancienne et dépassée : Pimentel, dans son rapport de 2003, a utilisé des données de Slesser et Lewis datant de 1979 pour évaluer l'énergie utilisée pour produire de l'acier, de l'acier inoxydable et du ciment ». Si des bases comme Ecoinvent (Frischknecht et al., 2004) actualisent probablement ces données, force est de constater que l'essentiel des actuelles ACV de biocarburants délimitent pour l'instant une frontière de système excluant les facteurs fixes de production agricoles et industriels (matériels, bâtiments, travailleurs permanents...). Cela pose question, notamment s'il s'agit de comparer ensuite les résultats avec des biocarburants produits au Sud où dominent des agricultures moins capitalistiques car beaucoup plus intensives en travail humain et animal. Dans ce dernier cas cependant (traction animale) se poseraient avec plus d'acuité encore les difficultés de calcul liées aux coproduits, ces derniers étant nombreux : production, parallèlement à la force de travail, de lait, de viande, de cuir, de bouses utilisées comme engrais ou combustible, etc. Autant de difficultés qui devraient être surmontées pour évaluer plus justement l'intérêt de biocarburants produits dans des contextes différents, au plan énergétique comme à celui des émissions en GES.

\section{L'émission de gaz à effet de serre}

Après une analyse standardisée de $6 \mathrm{ACV}$ d'éthanol de maïs américain, Farrell et al. (2006) concluent qu'à l'exception de travaux de Pimentel et Patzek, les autres études (celles de Graboski, Oliveira, Wang et Shapouri) montrent une moindre consommation d'énergie fossile par rapport à l'essence alors que l'émission de GES serait quasi identique. À ce dernier niveau, les biodiesels apparaissent bien plus avantageux, à côté de l'éthanol de canne brésilien et sa cogénération d'énergie (Tab. 3). Comme l'expliquent Hill et al. (2006), la filière biodiesel consomme non seulement moins d'énergie fossile émettrice de dioxyde de carbone $\left(\mathrm{CO}_{2}\right)$, notamment durant la phase de transformation industrielle (Tab. 2), mais également moins d'intrants agricoles tels que les engrais azotés qui libèrent du protoxyde d'azote $\left(\mathrm{N}_{2} \mathrm{O}\right)$ à pouvoir de réchauffement global 310 fois supérieur au $\mathrm{CO}_{2}$ (23 pour le méthane $-\mathrm{CH}_{4}$ ). Mais à cette moindre consommation d'intrants est aussi associée une moindre productivité de carburant par hec$\operatorname{tare}^{7}$ (Tab. 3) : un handicap certain (besoin de terres plus important pour produire autant) que certains relativisent : coproduction d'aliments pour animaux représentant autant d'espace gagné, pouvoir calorifique plus élevé des huiles, etc. Ce débat entre filières révèle une problématique centrale du changement climatique : 1 'intensification de l'agriculture augmente d'un côté les rendements de biomasse par hectare (photosynthèse plus importante, déforestation évitée...), mais restitue de l'autre des quantités croissantes de GES $\left(\mathrm{N}_{2} \mathrm{O}\right.$ en particulier, via les engrais azotés) auxquelles s'ajoutent généralement ensuite celles considérables de l'élevage (Steinfeld et al., 2006) qu'une partie de cette biomasse permet d'alimenter et de développer. Filières (végétales, animales) et itinéraires techniques (plus ou moins intensifs) ne peuvent donc être pensés indépendamment (CGGREF, 2006). En France, cette combinaison de filières et d'itinéraires conduit l'agriculture, formatée par un système alimentaire en aval et agroindustriel en amont, à une émission de GES représentant $17 \%$ des émissions nationales en 2003, voire $26 \%$ (devant les transports à $21 \%$ ) selon le type de comptabilité (CITEPA, $2005 a, b)$. Le résultat de cette combinaison mérite réflexion, réflexion transcendant largement celle aujourd'hui focalisée sur les possibilités de réduction de GES par les biocarburants.

Par delà ces considérations demeure une incertitude notoire sur les estimations de GES agricoles, spécialement de $\mathrm{N}_{2} \mathrm{O}$. Punter et al. (2004) et d'autres soulignent en effet que ces émissions de $\mathrm{N}_{2} \mathrm{O}$ impactent fortement le bilan GES global en raison du pouvoir de réchauffement global élevé (310) tout en variant grandement suivant le climat, le type de sol et bien sûr l'itinéraire

\footnotetext{
7 D'autant plus qu'il est impératif, pour des raisons agronomiques, d'alterner régulièrement les cultures oléagineuses avec d'autres.
} 
technique (Tab. 4). Trois grandes options se présentent aujourd'hui pour évaluer ces émissions : (i) extrapoler des résultats de mesures effectuées au champ dans le cadre d'itinéraires et de contextes agro-climatiques tous spécifiques, (ii) mobiliser un modèle biophysique alimenté par de telles mesures pour simuler d'autres situations, (iii) appliquer méthode et coefficients génériques fournis par le Groupe d'experts intergouvernemental sur l'évolution du climat - GIEC (IPCC, 1996, 2001b). Des auteurs ont comparé le résultat de ces options. Pour l'ADEME (2002) dont l'évaluation de base repose sur des données anglo-saxonnes (Skiba) provenant d'un nombre limité d'essais effectués avant 1996, l'option du GIEC conduit à augmenter significativement les émissions de GES des filières biocarburants : $+40-50 \%$ pour l'ester de colza ou tournesol, et $+10-20 \%$ pour l'éthanol de blé (pas de changement significatif avec la betterave). Mais CONCAWE (2004) affirme de son côté que la plage d'incertitude des résultats telle qu'indiquée par le GIEC (1996) peut être largement dépassée dans le cas de mesures au champ (jusqu'à 10 fois la valeur maximum indiquée par le GIEC). Son modèle GREASE conduit par ailleurs à des valeurs moyennes d'émission sensiblement supérieures à celles d'études basées sur l'option du GIEC, notamment en raison de simulations sur sols très riches en matières organiques. Enfin, par une approche très différente, elle aussi sujette à discussion, un prix Nobel de chimie (Crutzen et al., 2007) invite à réviser considérablement à la hausse les coefficients d'émission en $\mathrm{N}_{2} \mathrm{O}$ agricoles du GIEC. En attendant, puisque pratiques agricoles et contextes pédoclimatiques déterminent en grande partie le résultat des bilans, Farrell et al. (2006) ou encore Larson (2005) appellent à multiplier études et références en la matière plutôt que tergiverser sur quelques résultats.

Larson insiste également avec Delucchi (2004) sur l'importance d'intégrer les émissions de gaz à effet climatique indirect $\left(\mathrm{NO}_{x}, \mathrm{CO} \ldots\right)$ comme l'a par exemple tenté Kadam (2002) en Inde, et plus encore la dynamique du carbone des sols curieusement exclue de la très grande majorité des actuels écobilans de biocarburants. Cette dynamique est affectée par le changement d'occupation des terres (déstockage de carbone après mise en culture de forêts, de pâtures, voire de jachères) qui dépend lui-même des marchés et des politiques, mais aussi par les pratiques culturales, ce qui corrobore l'importance d'intégrer ces dernières dans les réflexions et prospectives relatives au climat. Des auteurs comme McCarl et Schneider (2001) ou Smith et al. (2007) s'y essayent même si l'éventail de données pour conduire de tels exercices demeure pour l'instant limité. Pour Lal (2004), la réduction des émissions de GES - tout comme le plus grand stockage de carbone dans les sols - passe d'abord par le non-labour, la gestion intégrée et économe d'engrais et de pesticides, l'irrigation au goutte-à-goutte ou souterraine (techniques économisant autant l'eau que l'énergie pour la pomper et la distribuer).

\section{Des controverses aux enjeux}

Comparer l'impact environnemental de produits concurrents (par exemple carburants fossiles/agricoles, éthanol/biodiesel...) à service rendu équivalent (par exemple kilomètre parcouru) n'est en pratique pas si aisé. Les écobilans s'y essayent et deviennent pour cela de curieux objets. D'un côté, et comme précédemment illustré, leur mise en œuvre et leurs conclusions s'avèrent dépendre non seulement du service considéré, mais aussi de co-services (coproduits) pouvant ou non être pris en compte, des objectifs poursuivis (comparaison de substituts, d'options technologiques, de sites de production...), $\mathrm{du}$ périmètre $\mathrm{d}$ 'analyse (systèmes plus ou moins limités verticalement et horizontalement...), de la nature et de l'étendue des indicateurs utilisés pour mesurer "l'impact environnemental » (consommation de ressources fossiles, effet de serre, biodiversité...), des bases de calcul, d'interprétation et de pondération de ces indicateurs. Cette relativité de méthodes et de résultats effraye et nécessite dans tous les cas d'afficher à chaque fois tous les éléments qui permettent de la juger et de la débattre. Trop techniciste pour certains (économistes...), trop simpliste pour d'autres (agronomes...), cet outil technico-économique de l'écologie industrielle est l'objet de multiples autres critiques (coût de réalisation, incertitude des résultats, etc.).

D'un autre côté cependant, la variabilité et la complexité des systèmes tant anthropiques qu'environnementaux ne peuvent être ignorées ou gommées, tout comme les besoins d'éco-évaluations ou d'éco-conceptions (Roux et al., 2005) de produits, services ou politiques. Les écobilans et leur multiplication peuvent alors apparaître comme de puissants moteurs pour tout à la fois (i) mobiliser et faire dialoguer des approches appartenant à des secteurs et des champs de connaissances très divers, (ii) générer des références et des questionnements là où il n'en existe actuellement pas ou peu, comme s'y attachent Basset-Mens (2005). C'est à ce moteur intégratif que nous aimons croire, dans le cadre d'une démarche visant à mieux comprendre et maitriser les univers techniques qui structurent les rapports entre les activités humaines et l'environnement biophysique naturel et construit. Dans cette perspective, la suite et fin de cet exposé s'attache à poursuivre et à élargir la discussion engagée sur les écobilans de biocarburants, autour de six points soulignant autant leurs limites actuelles que des perspectives de développement.

(1) Rares sont les écobilans de biocarburants étendant leur analyse à d'autres impacts environnementaux que l'émission directe de GES, et les quelques tentatives 
Tableau 4. Principaux facteurs d'émissions de GES par l'agriculture.

\begin{tabular}{|c|c|c|c|}
\hline Cadre d'inventaire du GIEC & $\begin{array}{l}\text { Facteurs } \\
\text { d'émission }\end{array}$ & $\begin{array}{l}\text { Émission } \\
\text { principale }\end{array}$ & Commentaires \\
\hline $\begin{array}{l}\text { Secteur « LULUCF » Utilisation des } \\
\text { terres, changement d'affectation des } \\
\text { terres et foresterie (IPCC, 2003) }\end{array}$ & $\begin{array}{l}\text { Mise en culture } \\
\text { de jachères, de } \\
\text { prairies } \\
\text { permanentes, de } \\
\text { forêts... }\end{array}$ & $\mathrm{C}$ & $\begin{array}{l}\text { - } \Delta \text { suivant climat et type de sol } \\
\text { - déstockage de } C \text { stocké dans le sol } \\
\text { - déstockage de } C \text { stocké dans la biomasse sou- } \\
\text { terraine et aérienne }\left(1 \mathrm{~m}^{3} \text { bois }=1 \text { tonne } \mathrm{CO}_{2}\right) \\
\text { - } \Delta \text { suivant l'usage et l'effet de substitution de } \\
\text { la biomasse extraite (bois d'œuvre, bois de } \\
\text { chauffe...) }\end{array}$ \\
\hline
\end{tabular}

\begin{tabular}{|c|c|c|c|}
\hline \multirow{5}{*}{$\begin{array}{l}\text { Secteur « Agriculture » } \\
\text { (GIEC, 2001b, chapitre 4) }\end{array}$} & $\begin{array}{l}\text { Brûlage de savane } \\
\text { ou déchets } \\
\text { agricoles }\end{array}$ & $\mathrm{CO}_{2}$ & \\
\hline & Culture de riz & $\mathrm{CH}_{4}$ & - \avec drainage \\
\hline & $\begin{array}{l}\text { Émissions des } \\
\text { sols }\end{array}$ & $\mathrm{N}_{2} \mathrm{O}$ & $\begin{array}{l}\text { - } \Delta \text { suivant climat et sol ( } \nearrow \text { avec épandage des } \\
\text { minéraux) } \\
\text { - } \nearrow \text { avec pâture d'animaux ou épandage de } \\
\text { fumure dynamique } \\
\text { - } \nearrow \text { avec épandage de résidus } \\
\text { - } \nearrow \text { avec culture de légumineuses }\end{array}$ \\
\hline & $\begin{array}{l}\text { Gestion des } \\
\text { déjections } \\
\text { animales }\end{array}$ & $\begin{array}{l}\mathrm{CH}_{4} \\
\mathrm{~N}_{2} \mathrm{O}\end{array}$ & $\begin{array}{l}\text { - } \nearrow \text { avec température extérieure } \\
\text { - } \Delta \text { suivant pratique (stockage liquide ou solide, } \\
\text { épandage journalier ou laissé sur pâture...) }\end{array}$ \\
\hline & $\begin{array}{l}\text { Fermentation } \\
\text { entérique }\end{array}$ & $\mathrm{CH}_{4}$ & - $\nearrow$ avec cellulose de la ration \\
\hline \multirow{3}{*}{$\begin{array}{l}\text { Secteurs «Énergie », « Industrie »... } \\
\text { ou hors cadre }\end{array}$} & $\begin{array}{l}\text { Utilisation } \\
\text { d'énergie fossile à } \\
\text { la ferme }\end{array}$ & $\mathrm{CO}_{2}$ & $\begin{array}{l}\text { - Traction, irrigation, moisson, séchage... } \\
\text { - Chauffage, réfrigération... }\end{array}$ \\
\hline & $\begin{array}{l}\text { Production des } \\
\text { facteurs de } \\
\text { production }\end{array}$ & $\begin{array}{l}\mathrm{CO}_{2} \\
\mathrm{~N}_{2} \mathrm{O}\end{array}$ & $\begin{array}{l}\text { - Engrais, pesticides, aliments pour animaux... } \\
\text { Énergie } \\
\text { - Bâtiments, Matériels... }\end{array}$ \\
\hline & Transports & $\mathrm{CO}_{2}$ & $\begin{array}{l}\text { - Marchandises, équipements et hommes } \\
\text { - En amont voire en aval de la phase de produc- } \\
\text { tion } \\
\text { - Local, national, international }\end{array}$ \\
\hline
\end{tabular}

Légende : les émissions de carbone $(\mathrm{C})$, dioxyde de carbone $\left(\mathrm{CO}_{2}\right)$, méthane $\left(\mathrm{CH}_{4}\right)$ ou protoxyde d'azote $\left(\mathrm{N}_{2} \mathrm{O}\right)$ répertoriées ou non comme «agricoles » par le GIEC (Groupe d'experts intergouvernemental sur l'évolution du climat) varient considérablement $(\Delta)$, diminuent $(\searrow$ ) ou augmentent $(\nearrow)$ suivant les éléments mentionnés.

dans ce sens, comme celles de l'ADEME (2002), Blottnitz et Curran (2007) ou Galbraith et al. (2006), se concluent sur beaucoup d'incertitudes ou d'inconnues, que ce soit en termes de consommation en eau ${ }^{8}$, de biodiversité, d'eutrophisation, d'acidification de la basse atmosphère,

8 Sur le sujet, voir également Bordet et Michez (2006) ou encore Roberts et al. (2007), Fraiture et al. (2008). de destruction de la couche d'ozone ou de pollution photochimique, de toxicité terrestre et aquatique, etc. Mais la phase agricole s'avère ici encore déterminante voire handicapante comparée aux filières fossiles (Zah et al., 2007), ceci alors que méthodes et références pour procéder aux diagnostics agro-environnementeaux demeurent incomplètes ou expérimentales (Bochu, 2002 ; 
Cunningham et al., 2003 ; Doublet, 2006 ; EEA, 2005 ; Frischknecht et al., 2004; Vilain, 2000, etc.).

(2) Le développement et l'affirmation de ces diagnostics permettraient pourtant - et aussi - de mieux arbitrer la valorisation des résidus de récolte. Leur utilisation à des fins énergétiques est en effet maintenant encouragée, notamment pour améliorer l'efficacité énergétique des filières actuelles d'éthanol de céréales ( $c f$. supra). Ceci nourrit diverses spéculations à l'échelle planétaire (De La Torre Urgate in Hazell et Pachauri, 2006 ; IEA, 2004, 140 ; WWI, 2006, 12) d'autant plus que les techniques de transformation des biomasses ligno-cellulosiques pourraient bientôt s'avérer plus performantes et rentables (biocarburants de seconde génération valorisant la plante entière) ${ }^{9}$. Lal (2005) avertit cependant : l'exportation systématique de toutes les biomasses aériennes hypothéquerait la qualité des sols puisque les résidus actuellement laissés sur champ favorisent le stockage de carbone organique, stimulent l'activité et la diversité des faunes souterraines, atténuent l'érosion ou les pollutions, concourent finalement à soutenir des rendements agricoles élevés et durables.

(3) Dans de nombreuses situations au Sud où l'on oublie fréquemment que la combustion de résidus de récolte par les ménages économise de l'énergie fossile, ces résidus servent également à alimenter les animaux, pour finalement fournir des produits et services (trait, lait, viande, œufs, cuir, laine, bouse, etc.) au rendement énergétique et à impact écologique peut-être inférieurs aux élevages industriels. Mais les différences de productivité par animal et l'actuelle restriction d'écobilans dans le domaine (Castellini et al., 2006; Meul et al., 2007 ; Mishra et Dikshit, 2004; Ogino et al., 2007...) ne permettent pas, là encore, de conclure. Elobeid et al. (2006) avertissent parallèlement qu'un développement accentué aux États-Unis des surfaces de maïs énergétique se fera au détriment des productions de porc et de volaille, ainsi que des exportations de tourteaux de soja qui alimentent désormais de nombreux élevages dans le monde. Les produits animaux deviendraient donc plus onéreux dans nos assiettes, ce qui amène une question posée par d'autres : peut-on vraiment nourrir correctement et durablement à la fois 9 milliards d'humains à l'horizon 2050 et au moins trois fois plus de têtes en animaux d'élevage ${ }^{10}$ (Collomb, 1999; Dorin et Landy, 2002 ; Griffon, 2006 ; MEA, 2005 ; Parmentier, 2007; Smil, 2000) ? Pour Pretty et al. (2005) ou encore Eshel et Martin (2006), nos modes occidentaux d'alimentation sont déjà coûteux pour le climat : aux États-Unis, un régime non végétarien émettrait $1485 \mathrm{~kg}$ en équivalent $\mathrm{CO}_{2}$ de plus par personne qu'un régime végétarien fournissant la

\footnotetext{
${ }^{9}$ Pour un exposé approfondi, voir Cormeau et Gosse, in Tréguer (2007).

10 Selon Faostat, le stock de ces animaux dépasserait 20 milliards de têtes en 2000, dont 3,3 de gros herbivores et/ou ruminants.
}

même quantité de calories, soit $6 \%$ des émissions nationales de GES. N'oublions pas cependant que ces animaux peuvent fournir d'autres services qu'alimentaires (traction, cuir, fertilisants, etc.) tout en valorisant des espaces comme les prairies dont on connaît la grande importance comme réservoir de carbone, ainsi que pour la biodiversité et l'eau.

(4) Malgré une hausse des coûts de production pour les éleveurs, le développement des biocarburants pourrait globalement profiter à l'agriculture, en retournant durablement la tendance baissière des prix à la ferme observée pendant plusieurs décennies à l'échelle internationale, mais aussi en créant des emplois dans le secteur. Pour la France cependant, le gain économique net du programme biocarburants n'est pas si évident pour Sourie et al. (2005) avec, pour l'agriculture, un surplus qui s'effondrerait dès que les cultures énergétiques s'étendraient des jachères aux terres actuellement affectées à des productions alimentaires. Pour les sociétés au Sud, le bilan économique pourrait être plus favorable, notamment parce que la production locale de biocarburants améliorerait l'accès à l'énergie des populations en zones rurales (De La Torre Urgate in Hazell et Pachauri, 2006 ; Schmidhuber, 2007). Mais ce bénéfice reste suspendu à diverses conditions (Dufey, 2006 ; Kojima et Johnson, 2005), notamment deux : (i) la non-captation des profits par de gros complexes agroindustriels, (ii) l'augmentation concomitante des productivités à l'hectare. À défaut, comme le simulent Rosegrant et al. (in Hazell et Pachauri, 2006) avec le modèle IMPACT de l'Institut international de recherche sur les politiques alimentaires (IFPRI), les conséquences sur la sécurité alimentaire des populations seraient dramatiques, tout spécialement en Afrique Subsaharienne.

(5) Cette perspective de conflit d'usage des terres entre alimentation et énergie inquiète aussi Rainelli (2007) même si la source ligno-cellulosique devient rentable. Inquiétude partagée par d'autres qui en viennent, pour cette raison et des considérations écologiques, à préconiser le développement des biocarburants sur les terres «marginales », de trop faible qualité de sol pour la production alimentaire, via des espèces herbacées ou arborées nécessitant peu d'intrants (Hill et al., 2006; Oliveira et al., 2005). D'après Tilman et ses collègues (2006), le rendement en bioénergie d'une combinaison de 18 espèces herbacées établies sur des sols peu fertiles ou dégradés serait, après une décennie, $238 \%$ supérieur à celui des actuelles productions de biocarburant, cela avec peu d'engrais et de traitements chimiques (cultures « LIHD » pour Low Input - High Diversity) et sans éroder la biodiversité des flores et faunes locales.

(6) Les cultures à base de lupin, pâturin, fétuque, pourghère (jatropha), miscanthus, panic (switchgrass) ou combinaison plus sophistiquée d'espèces végétales ne seront effectives sur des terres marginales que si leur 
rentabilité s'avèrent démontrée pour le paysan comme pour l'industriel dont le bassin d'approvisionnement ne peut être trop dispersé en raison des coûts de transport. Pour l'heure, c'est plutôt une expansion à grande échelle des cultures énergétiques les plus rentables qui s'organise : canne à sucre et soja en Amérique du Sud, huile de palme en Asie du Sud-Est. Cette dynamique au Sud encouragée par une demande solvable croissante de biocarburants du Nord ne peut à son tour qu'encourager une déforestation accrue des forêts tropicales, directement ou par effet domino. Or la conservation des forêts tropicales est un élément clef dans la préservation du système climatique (Gitz et Ciais, 2004 ; Stern, 2006). Pour le bilan carbone, au Brésil, la canne consacrée à la production d'éthanol éviterait d'émettre 2 tonnes de carbone par hectare et par an (plus de 4 selon Macedo, 1998) alors que la mise en culture d'une forêt tropicale déstocke 156 à 305 tonnes de carbone par hectare (forêt secondaire/primaire), et que l'afforestation permet de son côté d'emmagasiner 4 à 8 tonnes de carbone par hectare et par an (Righelato et Spracklen, 2007).

\section{Conclusion}

Les actuels écobilans de biocarburants permettent de poser un ensemble de questions soulevées par l'émergence et la possible grande extension de ces productions énergétiques. Ces questions sont nombreuses et profondément complexes. Elles le seraient bien moins si ces mêmes écobilans parvenaient à dépasser : (i) des divergences d'ordre méthodologique et des difficultés d'accès aux données de base qui ne facilitent ni la comparaison des résultats, ni leur appropriation par d'autres, alors que les économistes - en particulier - gagneraient à les intégrer dans leur raisonnement (Delucchi, 2004); (ii) leur manque de représentativité dû à leur nombre encore insuffisant. Cette double restriction est inquiétante s'il s'agit d'identifier ou d'imaginer des systèmes durables de productions bioénergétiques au Nord comme au Sud de la planète, puisque le résultat des écobilans (efficacité énergétique, émissions de GES, etc.) s'avère fortement dépendre, d'une part des techniques agricoles employées (motorisation, intrants chimiques, irrigation) dans des écosystèmes donnés (types de climat et de sol), d'autre part d'arbitrages relatifs à la valorisation des coproduits (production animale, qualité des sols, production d'énergie). Il serait donc bénéfique d'élargir grandement ces actuels écobilans à d'autres contextes, biomasses et modes agricoles de production.

Enfin, si les actuelles productions occidentales de biocarburants apparaissent encore peu performantes aux plans énergétique et climatique (en particulier celles issues de cultures céréalières motorisées et utilisant des niveaux élevés d'intrants chimiques), il faut leur reconnaître un mérite indéniable : celui d'avoir suscité dans le secteur agricole des analyses et réflexions en termes d'écobilans. C'était urgent. Il reste encore beaucoup à faire dans le domaine pour mieux penser, sous diverses contraintes pédoclimatiques et économiques, ici et là dans le monde, les productions à venir de biomasses alimentaires et non alimentaires. Ce dernier demi-siècle de «modernisations » ou de « révolutions vertes » agricoles, basé sur une énergie fossile bon marché et une quasi-absence de préoccupation écologique appelle de profondes réformes. Il s'agit $\mathrm{d}$ 'inventer (ou de redécouvrir et d'améliorer) des agricultures ou des agro-foresteries qui, dans leur écosystème respectif, et par des combinaisons judicieuses d'espèces végétales et animales, permettent d'extraire et de valoriser durablement les plus grandes quantités possibles de biomasses.

\section{Références}

ADEME, DIREM, Ecobilan et al., 2002. Bilans énergétiques et gaz à effet de serre des filières de production de biocarburants en France. Note de synthèse, Paris, Agence de l'Environnement et de la Maîtrise de l'Énergie.

AI, 2006. A High Growth Strategy for Ethanol, Report of an Aspen Institute Policy Dialogue, Washington DC, The Aspen Institute.

Basset-Mens, C., 2005. Propositions pour une adaptation de l'Analyse de Cycle de Vie aux Systèmes de production agricole, Thèse de doctorat, Agrocampus Rennes, INRA, Rennes.

Blottnitz, H.V., Curran, M.A., 2007. A review of assessments conducted on bio-ethanol as a transportation fuel from a net energy, greenhouse gas, and environmental life-cycle perspective, Journal of Cleaner Production, 15, 7, 607-619.

Bochu, J.-L., 2002. PLANETE : Méthode pour l'analyse énergétique de l'exploitation agricole et l'évaluation des émissions de gaz à effet de serre, Toulouse, Solagro.

Bordet, J., Michez, J.-M., 2006. Mise en œuvre du plan biocarburant au regard de la protection de la resource en eau, Rapport CGAAER nº 15, IGE/05/055, Inspection générale de l'environnement.

Castellini, C., Bastianoni, S., Granai, C., et al., 2006. Sustainability of poultry production using the emergy approach: Comparison of conventional and organic rearing systems, Agriculture, Ecosystems and Environment, 114, 343-350.

CES, 2004. Les débouchés non alimentaires des produits agricoles : un enjeu pour la France et l'Union Européenne, Rapport présenté au nom de la Section de l'Agriculture et de l'Alimentation par Jean-Claude Pasty, Paris, Conseil économique et social.

CGGREF, 2006. Gaz à effet de serre, Bioénergies, Agriculture et Forêt : quels enjeux ?, CGGREF infos, Conseil général du génie rural, des eaux et des forêts.

CITEPA, 2005a. Inventaire des émissions de gaz à effet de serre en France au titre de la convention cadre des Nations unies sur les changements climatiques, Rapport national d'inventaire, Paris, Centre interprofessionnel technique d'études de la pollution atmosphérique.

CITEPA, 2005b. Inventaire des émissions de polluants atmosphériques en France. Secten, Rapport d'inventaire national, Paris, Centre interprofessionnel technique d'études de la Pollution Atmosphérique. 
Collomb, P., 1999. Une voie étroite pour la sécurité alimentaire d'ici à 2050, Paris, Economica.

CONCAWE, 2002. Energy and greenhouse gas balance of biofuels for europe - an update, Report 2/02, Brussels, CONCAWE Ad Hoc Group on Alternative Fuels.

CONCAWE, 2004. Well-to-Wheels analysis of future automotive fuels and powertrains in the European context, Well to Wheels Report Version 1b, Brussels, CONCAWE, EUCAR, JRC.

Crutzen, P.J., Mosier, A.R., Smith, K.A., et al., 2007. $\mathrm{N}_{2} \mathrm{O}$ release from agro-biofuel production negates global warming reduction by replacing fossil fuels, Atmospheric Chemistry and Physics Discussions, 7, 11191-11205.

Cunningham, B., Battersby, N., Wehrmeyer, W., et al., 2003. A Sustainability Assessment of a Biolubricant, Journal of Industrial Ecology, 7, 3-4, 179-192.

Delucchi, M.A., 2004. Conceptual and Methodological Issues in Lifecycle Analyses of Transportation Fuels, Report UCD-ITSRR-04-45 prepared for the The US Environmental Protection Agency Office of Transportation and Air Quality, Institute of Transportation Studies.

Dorin, B., Landy, F., 2002. Agriculture et alimentation de l'Inde. Les vertes années (1947-2001), Espaces ruraux, Paris, INRA éditions.

Doublet, S., 2006. DIALECTE. Diagnostic Agri-environnemental d'exploitation, Toulouse, Solagro.

Dufey, A., 2006. Biofuels production, trade and sustainable development: emerging issues, Sustainable Markets Discussion Paper 2, London, International Institute for Environment and Development.

EEA, 2005. Agriculture and environment in EU-15. The IRENA indicator report, Copenhagen, European Environmental Agency.

Elobeid, A., Tokgoz, S., Hayes, D.J., et al., 2006. The long-run impact of corn-based ethanol on the grain, oilseed, and livestock sectors: A preliminary assessment, CARD Briefing Paper, 06-BP 49, 1-16.

Elsayed, M.A., Matthews, R., Mortimer, N.D., 2003. Carbon and energy balances for a range of biofuels options, Report Project B/B6/00784/REP, URN 03/386, Sheffield, Sheffield Hallam University.

Eshel, G., Martin, A., 2006. Diet, Energy, and Global Warming, Earth Interactions, 10, 9, 1-17.

Farrell, A.E., Plevin, R.J., Turner, B.T., et al., 2006. Ethanol can contribute to energy and environmental goals, Science, 311, 506-508.

Fernandes, S.D., Trautmann, N.M., Streets, D.G., et al., 2007. Global biofuel use, 1850-200, Global Biogeochemical Cycles, 21, GB2019, 1-15.

Fraiture, C.D., Giordano, M., Yongsong, L., 2008. Biofuels and implications for agricultural water use: blue impacts of green energy, Water Policy, 10, 51, 67-81.

Frischknecht, R., Althaus, H.-J., Doka, G., et al., 2004. Ecoinvent 2000. Overview and Methodology, Final report 1, Duebendorf, Swiss Centre for Life Cycle Inventories.

Galbraith, D., Smith, P., Mortimer, N. et al., 2006. Review of greenhouse gas life cycle emissions, air pollution impacts and economics of biomass production and consumption in Scotland, Environmental Research Report SEERAD Project FF/05/08, Edinburgh, Scottish Executive Environment and Rural Affairs.

Gitz, V., Ciais, P., 2004. Future expansion of agriculture and pasture acts to amplify atmospheric $\mathrm{CO}_{2}$ levels in response to fossil-fuel and land-use change emissions, Climatic Change, 67, 2-3, 161-184.
GM, 2002. GM well-to-wheel analysis of energy use and greenhouse gas emissions of advanced fuel/vehicle systems. A european study, Full Background Report, Ottobrunn, GM, BP, ExxonMobil, TotalFinaElf.

GMC, ANL, BP et al., 2001. Well-to-wheel energy use and greenhouse gas emissions of advanced fuel/Vehicle systems. North American analysis, General Motors Corporation, Argonne National Laboratory, BP, ExxonMobil, Shell.

Graboski, M.S., 2002. Fossil energy use in the manufacture of corn ethanol, Report prepared for the National Corn Growers Association, St. Golden, Colorado School of Mines.

Griffon, M., 2006. Nourrir la planète. Pour une révolution doublement verte, Paris, Odile Jacob.

Hazell, P., Pachauri, R.K., 2006. Bioenergy and Agriculture: Promises and Challenges, 2020 Focus 14, IFPRI, TERI, Washington DC.

Hecht, L., 2007. Smell of gigantic hoax in government ethanol promotion, Executive Intelligence Review, 21-26.

Hill, J., Nelson, E., Tilman, D., et al., 2006. Environmental, economic, and energetic costs and benefits of biodiesel and ethanol biofuels, PNAS, 103, 30, 11206-10.

IEA, 2004. Biofuels for Transport - An International Perspective, Paris, International Energy Agency.

IPCC, 1996. Revised 1996 IPCC Guidelines for national greenhouse gas inventories. Reference Manual (Vol. 3), Intergovernmental Panel on Climate Change, http://www.ipcc-nggip.iges.or.jp/ public/gl/invs6.htm

IPCC, 2001a. Climate Change 2001: Mitigation, Cambridge, Cambridge University Press.

IPCC, 2001b. Good practice guidance and uncertainty management in national greenhouse gas inventories, Intergovernmental Panel on Climate Change, http://www.ipcc-nggip.iges.or.jp/public/ gp/french/gpgaum_fr.htm

IPCC, 2003. Good practice guidance for land use, Land-use change and forestry, Intergovernmental Panel on Climate Change, http://www.ipcc-nggip.iges.or.jp/public/gpglulucf/ gpglulucf_contents.htm

Jeuland, N., Montagne, X., Gautrot, X., 2004. Potentiality of ethanol as a fuel for dedicated engine, Oil $\mathcal{E}$ Gas Science and Technology, 59, 6, 559-570.

Kadam, K.L., 2002. Environmental benefits on a life cycle basis of using bagasse-derived ethanol as a gasoline oxygenate in India, Energy Policy, 30, 371-384.

Kavanagh, E., 2006. Looking at biofuels and bioenergy, Science, 312, 23, 1743-1748.

Kojima, M., Johnson, T., 2005. Potential for biofuels for transport in developing countries, ESMAP Reports, Washington DC, World Bank.

Lal, R., 2004. Carbon emission from farm operations, Environment International, 30, 981-990.

Lal, R., 2005. World crop residues production and implications of its use as a biofuel, Environment International, 31, 575-584.

Larson, E.D., 2005. Liquid biofuel systems for the transport sector: a Background Paper, GEF/STRAP Workshop on Liquid Biofuels, New Delhi, 29 August - 1 September.

Macedo, I.d.C., 1998. Greenhouse gas emissions and energy balances in bio-ethanol production and utilization in Brazil (1996), Biomass and Bioenergy, 14, 1, 77-81.

Macedo, I.d.C., Leal, M.R.L.V., Silva, J.E.A.R.d., 2002. Greenhouse gas (GHG) emissions in the production and use of ethanol Brazil: Present Situation (2002), NIPE, Campinas.

McCarl, B.A., Schneider, U.A., 2001. Greenhouse gas mitigation in US agriculture and forestry, Science, 294, 2481-2482. 
MEA, 2005. Ecosystems and human well-being: Synthesis, Millennium Ecosystem Assessment, Whashington DC, World Resources Institute.

Meul, M., Nevens, F., Reheul, D., et al., 2007. Energy use efficiency of specialised dairy, arable and pig farms in Flanders, Agriculture, Ecosystems and Environment, 119, 135144.

Mishra, S.N., Dikshit, A.K., 2004. Environment and Livestock in India. With a comparative study of the indian and US dairy system, New Delhi, Manohar.

Ogino, A., Orito, H., Shimada, K., et al., 2007. Evaluating environmental impacts of the Japanese beef cow-calf system by the life cycle assessment method, Animal Science Journal, $78,424-432$.

Oliveira, M.E.D.D., Vaughan, B.E., Rykiel, E.J., 2005. Ethanol as fuel: energy, carbon dioxide balances, and ecological footprint, BioScience, 55, 7, 593-602.

Parmentier, B., 2007. Nourrir l'humanité. Les grands problèmes de l'agriculture mondiale au XXI' siècle, Paris, La Découverte.

Pimentel, D., 2001. Biomass utilization, Limits of, in Encyclopedia of Physical Science and Technology, Academic Press, 13.

Pimentel, D., Patzek, T., 2007. Ethanol production: energy and economic issues related to US and Brazilian sugarcane, Natural Resources Research, 16, 3, 235-242.

Pimentel, D., Patzek, T.W., 2005. Ethanol production using corn, switchgrass, and wood; biodiesel production using soybean and sunflower, Natural Resources Research, 14, 1, 65-76.

Pretty, J.N., Ball, A.S., Lang, T., et al., 2005. Farm costs and food miles: An assessment of the full cost of the UK weekly food basket, Food Policy, 30, 1-19.

Punter, G., Rickeard, D., Larivé, J.-F., et al., 2004. Well-to-wheel evaluation for production of ethanol from wheat, Report FWG-P04-024, Low Carbon Vehicle Partnership.

Rainelli, P., 2007. L'avenir des biocarburants et incidences sur l'équilibre des marchés agricoles, Policy Paper 25, Paris, Notre Europe.

Richards, I.R., 2000. Energy balances in the growth of oilseed rape for biodiesel and of wheat for bioethanol, Levington Agriculture Report, Suffolk, British Association for Bio Fuels and Oils.

Righelato, R., Spracklen, D.V., 2007. Carbon mitigation by biofuels or by saving and restoring forests?, Science, 317, 907, 902.

Roberts, M.G., Male, T.D., Toombs, T.P., 2007. Potential impacts of biofuels expansion on natural resources. A case study of the ogallala aquifer region, New York, Environmental Defence.

Roux, P., Patingre, J.-F., Giroux, F., et al., 2005. Ecotechnologies et éconconception : concepts et mise en œuvre, Ingénieries, 42, 55-70.

Sadones, P., 2006. Les agrocarburants, Rapport EDEN 2006, Yvetot, Énergie durable en Normandie.

Schmidhuber, J., 2007. Biofuels: An emerging threat to Europe's Food Security?, Policy Paper, Paris, Notre Europe.
Shapouri, H., 2002. The Energy Balance of Corn Ethanol: An Update, Washington DC, United States Department of Agriculture.

Shapouri, H., 2004. The 2001 net energy balance of corn-ethanol (Preliminary), http://www.bioproducts-bioenergy.gov/pdfs/ net $\mid \% 20$ energy $\backslash \% 20$ balance.pdf

Sims, R.E.H., Hastings, A., Schlamadinger, B., et al., 2006. Energy crops: current status and future prospects, Global Change Biology, 12, 1-23.

Smeets, E.M.W., Faaij, A.P.C., 2007. Bioenergy potentials from forestry in 2050. An assessment of the drivers that determine the potentials, Climatic Change, 81, 353-390.

Smeets, E.M.W., Faaij, A.P.C., Lewandowski I.M. et al., 2007. A bottom-up assessment and review of global bio-energy potentials to 2050, Progress in Energy and Combustion Science, 33, 56-106.

Smil, V., 2000. Feeding the world. A Challenge for the twenty-first century, Cambridge, MIT Press.

Smith, P., Martino, D., Cai, Z., et al., 2007. Policy and technological constraints to implementation of greenhouse gas mitigation options in agriculture, Agriculture, Ecosystems and Environment, 118, 6-28.

Sourie, J.C., Tréguer, D., Rozakis, S., 2005. L'ambivalence des filières biocarburants, INRA Sciences Sociales, 20, 2, 1-8.

Steinfeld, H., Gerber, P., Wassenaar, T., et al., 2006. Livestock's long shadow environmental issues and options, Rome, Food and Agricultural Organization of the United Nations.

Stern, N., 2006. Stern Review on the economics of climate change, HM Treasuary, London, Cabinet Office.

Tilman, D., Hill, J., Lehman, C., 2006. Carbon-negative biofuels from low-input high-diversity grassland biomass, Science, 314, 1598-1600.

Tréguer, D. (Dir.), 2007. L'avenir à moyen terme des biocarburants : enjeux politiques, économiques, environnementaux et technologiques, Déméter 2008, Paris, Club Déméter, 97-302.

Vilain, L. (Dir.), 2000. La méthode IDEA. Indicateurs de durabilité des exploitations agricoles. Guide d'utilisation, Dijon, Educagri éditions.

WADE, 2004. Bagasse cogeneration. Global review and potential, Report, World Alliance for Decentralized Energy.

Wang, M., 2005. Updated energy and greenhouse gas emission results of fuel ethanol, The 15th International Symposium on Alcohol Fuels, San Diego, 26-28 September.

Woods, J., Bauen, A., 2003. Technology status review and carbon abatement potential of renewable transport fuels in the $U K$, Report B/U2/00785/REP, URN 03/982, London, Department of Trade and Industry.

WWI, 2006. Biofuels for transportation. Global potential and implications for sustainable agriculture and energy in the 21st century. Extented summary, Washington DC, Worldwatch Institute.

Zah, R., Böni, H., Gauch, M. et al., 2007. Ökobilanz von Energieprodukten, Ökologische Bewertung von Biotreibstoffen, Schlussbericht, Empa, St. Gallen.

Reçu le 5 décembre 2007. Accepté le 26 juin 2008. 